Check for updates

Cite this: Chem. Sci., 2019, 10, 6791

¿ All publication charges for this article have been paid for by the Royal Society of Chemistry

\section{Interplay between spin-crossover and luminescence in a multifunctional single crystal iron(II) complex: towards a new generation of molecular sensors $\uparrow$}

\author{
Bouabdellah Benaicha, ${ }^{a}$ Khanh Van Do, ${ }^{b}$ Aymen Yangui, (DD ${ }^{c}$ Narsimhulu Pittala, ${ }^{a}$ \\ Alain Lusson, ${ }^{\mathrm{b}}$ Mouhamadou Sy, (D) ${ }^{\mathrm{b}}$ Guillaume Bouchez, ${ }^{\mathrm{b}}$ Houcem Fourati, (D) ${ }^{\mathrm{b}}$ \\ Carlos J. Gómez-García, (D) ${ }^{d}$ Smail Triki (D) *a and Kamel Boukheddaden (D) *b
}

\begin{abstract}
We present a new example of a mononuclear iron(॥) complex exhibiting a correlated spin-crossover (SCO) transition and strong fluorescence, whose coordination sphere is saturated, for the first time, by six phosphorescent ligands. The interplay between SCO and light emission properties in the thermal region of the spin transition was investigated by means of magnetic, fluorescence, optical absorption and optical microscopy measurements on a single crystal. Overall, the results show an excellent correlation between fluorescence and magnetic data of the present gradual transition, indicating an extreme sensitivity of the optical activity of the ligand to the spin state of the active iron(II) ions. These results open the way for conceiving new prototypes of pressure and temperature sensors based on this synergy between SCO and luminescence properties. In particular, the fact that cooperative SCO material is not a prerequisite for obtaining such synergetic effects, is useful for the design of thin films or nanoparticles, in which the cooperativity is reduced, for appropriate implementation in nanosized devices to enhance the sensing properties at the nanoscale.
\end{abstract}

Received 13th May 2019

Accepted 6th June 2019

DOI: $10.1039 / c 9 s c 02331 c$

rsc.li/chemical-science

\section{Introduction}

Among the switchable molecular solids, spin crossover (SCO) complexes are interesting specifically because of their multifunctional properties at the molecular scale and they are used as nano-switches due to many assets compared to classic silicon technology. Hence, SCO solids have potential applications in the development of new generations of electronic devices. Indeed, iron(II)-based SCO complexes were selected due to their variety of nano-structuration possibilities ${ }^{1}$ which will be useful for implementation into devices for a set of currently considered applications, e.g. as displays and memory devices, molecular sensors, and rapid switches, ${ }^{2}$ in addition to their

${ }^{a}$ Univ. Brest, CNRS, CEMCA, 6 Avenue Victor Le Gorgeu, C. S. 93837, 29238 Brest Cedex 3, France. E-mail: Smail.Triki@univ-brest.fr

${ }^{b}$ Groupe d'Etude de la Matière Condensée, CNRS UMR8635, Université de Versailles Saint Quentin, Université Paris-Saclay, 45 Avenue des Etats-Unis, 78035 Versailles cedex, France.E-mail: Kamel.boukheddaden@uvsq.fr

'Department of Chemistry and Biochemistry, University of Oklahoma, 101 Stephenson Parkway, Norman, OK 73019, USA

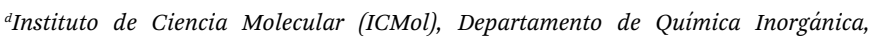
Universidad de Valencia, C/Catedrático José Beltrán 2, 46980 Paterna, Spain

$\dagger$ Electronic supplementary information (ESI) available. CCDC 1565194 and 1565195. For ESI and crystallographic data in CIF or other electronic format see DOI: $10.1039 / \mathrm{c} 9 \mathrm{sc} 02331 \mathrm{c}$ barocaloric properties for refrigeration which have been recently demonstrated. ${ }^{3}$ The SCO phenomenon, mostly in iron(II) systems, is an entropy-driven process involving the switching between the diamagnetic low-spin (LS, $S=0,{ }^{1} \mathrm{~A}_{1 \mathrm{~g}}$ ) and the paramagnetic high-spin (HS, $S=2,{ }^{5} \mathrm{~T}_{2 \mathrm{~g}}$ ) states, as a response to an external stimulus as a variation of temperature, pressure, light or a magnetic field, ${ }^{1 a}$ as well as by an electric polarization leading to magneto-electric effects. ${ }^{4}$ As a result, different optical, magnetic, vibrational and structural properties can be recorded by a variety of physical techniques. Among the challenging quests in multi-functional materials, the combination of the SCO phenomenon with luminescence in a synergetic way will extend the innovative nature of the SCO materials to new potential applications such as magneto-optical switches or new generation of multi-sensors. ${ }^{5-8}$

The attempts to achieve this goal are based on two strategies: the first one consists in the synthesis of non molecular composites combining the suited properties such for SCOluminescent core-shell nanoparticles ${ }^{9}$ or electroluminescent thin films doped by SCO complexes ${ }^{7}$ while the second strategy is based on the design of fluorescent SCO complexes in which the fluorophore groups act as metal complexes or organic counter anions ${ }^{5 a, 7 a, 10}$ or are organic molecules covalently linked to the SCO complexes. ${ }^{6,8 a, 11}$ While some materials, prepared using these strategies exhibit the two desired properties, ${ }^{5 a, 6-11}$ the 
correlation between the SCO transition and the fluorescence has been rarely observed..$^{\mathbf{6}-\boldsymbol{d}, \mathbf{8 , 9 , 1 1}}$ Among which those based on covalently linked fluorophore groups seem to be more promising multifunctional systems, since the corresponding, more rigorous synthetic approach, allows obtaining single crystals suitable for structural characterizations. Within this class of molecular systems, the first fluorescent SCO material was reported in 2011 by Garcia et al. ${ }^{6 a}$ as a dinuclear complex based on the $N$-salicylidene-4-amino-1,2,4-triazole ligand which is well-known for its strong fluorescence at the solid state. This material exhibits an abrupt SCO transition that can be tracked through the variation of the fluorescence signal. Using an original synthetic approach, J. Tao et al. ${ }^{6 \boldsymbol{b}}$ have reported in 2015 two fluorescent one-dimensional SCO coordination polymers, based on 3-amino-1,2,4-triazole ligand for which some of the triazole motifs were statistically grafted by the 1-pyrenecarboxaldehyde or rhodamine fluorophores. The two systems showed correlation between the thermally-induced SCO transition and associated fluorescence variation. Months later, the same group has reported another dinuclear $\mathrm{Fe}(\mathrm{II})$, almost similar to that described by Garcia group, which displayed three-step SCO behavior with multiple correlations with the fluorescence. ${ }^{6 c}$ More recently, B. Weber et al. ${ }^{11 a}$ and H.-Z. Kou et al. ${ }^{11 b}$ have separately designed, respectively, a SCO Fe(II) coordination polymer and a SCO discrete Fe(II) mononuclear complex, with thermal hysteresis loops above room temperature (RT) both exhibiting fluorescent properties with strong correlations between the light emission and the spin state properties. Nevertheless, besides the two dinuclear Fe(II) complexes which have been crystallographically characterized, ${ }^{\boldsymbol{b}, \boldsymbol{c}} \boldsymbol{c}$ the molecular structures of all these systems were assessed with essentially elemental analysis and X-ray powder diffraction. In addition, in none of these materials the coordination sphere was saturated by six fluorescent ligands. In this context, here we report on the mononuclear Fe(II) SCO and fluorescent complex [Fe(naphtrz $\left.)_{6}\right](\text { tcsme })_{2} \cdot 4 \mathrm{CH}_{3} \mathrm{CN}, \mathbf{C 1}$ (naph-trz $=\mathbf{L 1}=N$-(1,2,4-triazol-4-yl)1,8-naphthalimide; $\quad\left(\right.$ tcnsme $^{-}=1,1,3,3$-tetracyano-2thiomethylpropenide anion), whose coordination sphere is saturated, for the first time, by six phosphorescent ligands showing a coupling between the two properties. Due to this interaction, the emerging response is more than the sum of the of the individual responses of the ligand and the SCO metal. The fluorescence and the magnetic responses reveal the interplay between the SCO and the light emission properties in the thermal region of the SCO transition. In order to identify the interaction between the phosphorescent ligand and the spin transition of the $\mathrm{Fe}(\mathrm{II})$ active center, fluorescence studies have been also performed on the ligand naph-trz (L1). Furthermore, to assess the influence of the spin transition on the light emission properties in complex $\mathbf{C 1}$, the homologous compound $[\mathrm{Cu}$ (naph-trz) 6$](\text { tcnsme })_{2} \cdot 4 \mathrm{CH}_{3} \mathrm{CN}$ (C2) was synthesized and its thermal properties were investigated.

\section{Results and discussion}

The napht-trz molecule (L1) and the potassium salt K(tcnsme) were prepared according to modified synthetic strategies (see
ESI $\dagger) \cdot{ }^{12,13}$ Single crystals of $\left[\mathrm{M}(\text { napht-trz })_{6}\right](\text { tcnsme })_{2} \cdot 4 \mathrm{CH}_{3} \mathrm{CN}$ $\left(\mathrm{M}=\mathrm{Fe}^{\mathrm{II}}(\mathbf{C} 1) ; \mathrm{M}=\mathrm{Cu}^{\mathrm{II}}(\mathbf{C} 2)\right)$ have been prepared under aerobic conditions by refluxing acetonitrile solutions containing $\left[\mathrm{M}\left(\mathrm{BF}_{4}\right)_{2}\right] \cdot x \mathrm{H}_{2} \mathrm{O}\left(\mathrm{M}=\mathrm{Fe}^{\mathrm{II}}(\mathbf{C} 1) ; \mathrm{M}=\mathrm{Cu}^{\mathrm{II}}(\mathbf{C} 2)\right)$, napht-trz and $\mathrm{K}$ (tcnsme) in a $1: 6: 2$ molar ratio. Details of the synthesis, NMR spectra (Fig. S1-7†), Infrared spectra (Fig. S8-12†), as well as the relevant crystallographic parameters and data (Fig. S1315, Tables S1 and 2) are reported in the ESI. $\dagger$

The thermal variation of the product of the molar magnetic susceptibility per Fe(II) ion times the temperature $\left(\chi_{\mathrm{m}} T\right)$ shows at RT a value of $3.9 \mathrm{~cm}^{3} \mathrm{~K} \mathrm{~mol}^{-1}$ (Fig. 1a). This value is close to the expected one for an isolated HS $(S=2)$ hexacoordinated $\mathrm{Fe}(\mathrm{II})$ ion with $g \approx 2.28$. Upon cooling, the $\chi_{\mathrm{m}} T$ product remains constant down to $180 \mathrm{~K}$ and below this temperature, it shows a gradual decrease to reach a plateau of $c a .0 .45 \mathrm{~cm}^{3} \mathrm{~K} \mathrm{~mol}^{-1}$ below $c a .70 \mathrm{~K}$, indicating the presence of a gradual and almost complete HS to LS SCO with a $T_{1 / 2}=114 \mathrm{~K}$, measured as the maximum of the $\delta\left(\chi_{\mathrm{m}} T\right) / \delta T$ vs. $T$ plot (inset in Fig. 1a). At low temperatures the $\chi_{\mathrm{m}} T$ value indicates that there is a fraction of
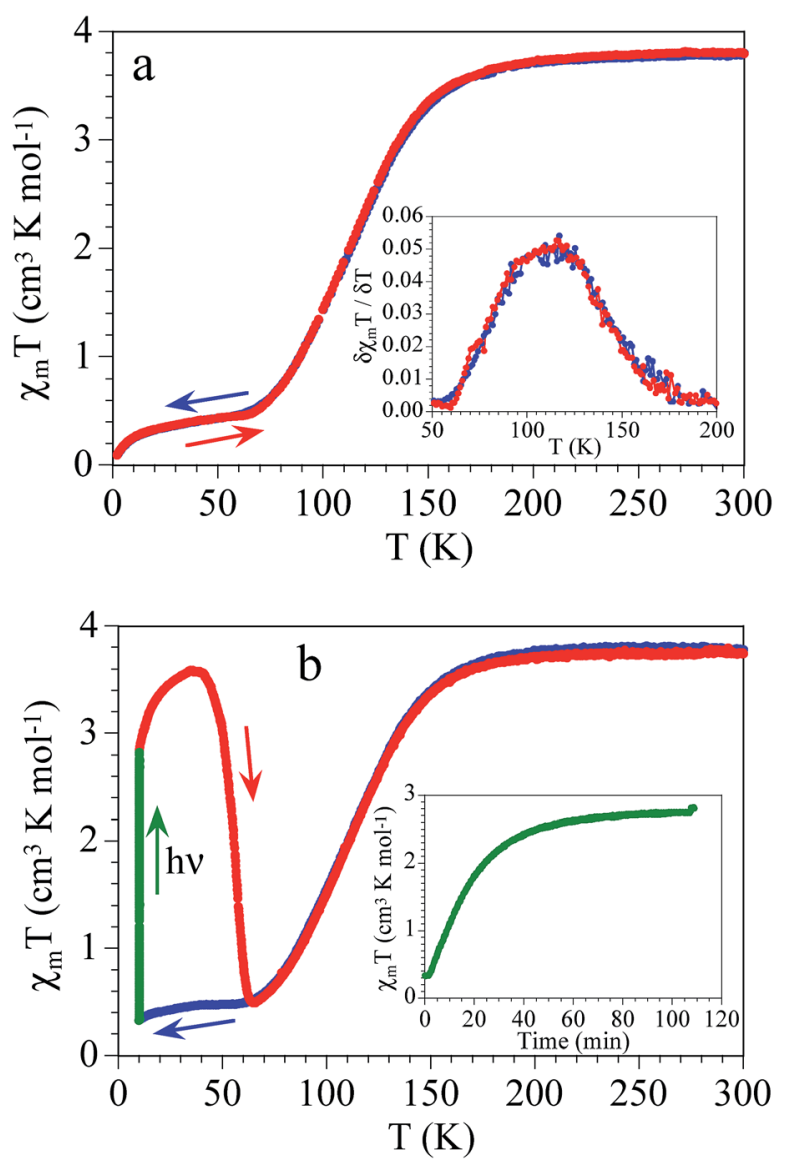

Fig. 1 (a) Thermal variation of the $\chi_{m} T$ product of [Fe(naph-trz) 6 ](tcnsme $)_{2} \cdot 4 \mathrm{CH}_{3} \mathrm{CN}$ (C1) in a cooling and heating scan in the thermal interval 2-300 K. Inset shows the thermal variation of $\frac{\partial\left(\chi_{m} T\right)}{\partial T}$. (b) Photo-magnetic measurements of $\mathrm{C} 1$ : dark cooling (blue points), irradiation at $10 \mathrm{~K}$ (green points) and dark heating after irradiation (red points). Inset shows the time evolution of the signal at $10 \mathrm{~K}$ under irradiation. Temperature scan rate is $0.4 \mathrm{~K} \mathrm{~min}^{-1}$. 
ca. $10 \%$ of the molecules that remains in the HS state. This fraction shows the expected zero field splitting, responsible of the more abrupt decrease of $\chi_{\mathrm{m}} T$ at lower temperatures (below $10 \mathrm{~K})$.

The photomagnetic measurements performed at $10 \mathrm{~K}$ show a continuous increase of the magnetization (Fig. 1b) when the sample is irradiated with a green laser $(532 \mathrm{~nm})$ to reach saturation after ca. 2 hours (inset of Fig. 1b). The saturation value is $2.8 \mathrm{~cm}^{3} \mathrm{~K} \mathrm{~mol}^{-1}$, indicating an almost complete recovery of the HS state. Indeed, when the sample is heated in dark, $\chi_{\mathrm{m}} T$ shows a gradual increase to reach a maximum value of $3.7 \mathrm{~cm}^{3} \mathrm{~K} \mathrm{~mol}^{-1}$ at $40 \mathrm{~K}$, characteristic of a magnetic response with zero a field splitting (magnetic anisotropy) which lifts the degeneracy of the spin state $S=2$, confirming the total photo-conversion to the HS state of C1. At higher temperature, the HS fraction relaxes back to the LS state at $T_{\text {LIESST }}=55 \mathrm{~K}$ (Fig. 1b). On further heating, the sample recovers the equilibrium curve with a gradual SCO temperature at $T_{1 / 2}=114 \mathrm{~K}$, as observed in Fig. 1a.

Based on the previous magnetic measurements, the crystal structure of $\mathbf{C 1}$ has been determined at $296 \mathrm{~K}$ and $100 \mathrm{~K}$ (see

Table 1 Fe-N distances $(\AA \AA)$, distortion parameters $\left(^{\circ}\right)$ in the HS and LS states. $\Sigma$ is the sum of the deviation from $90^{\circ}$ of the 12 cis-angles of the $\mathrm{FeN}_{6}$ octahedron $^{14}$

\begin{tabular}{lll}
\hline$T(\mathrm{~K})$ & $296(\mathrm{HS})$ & $100(\mathrm{LS})$ \\
$\mathrm{Fe} 1-\mathrm{N} 1$ & $2.200(4)$ & $1.999(4)$ \\
$\mathrm{Fe} 1-\mathrm{N} 2$ & $2.190(4)$ & $1.993(4)$ \\
$\mathrm{Fe} 1-\mathrm{N} 3$ & $2.176(4)$ & $1.994(4)$ \\
$\langle\mathrm{Fe}-\mathrm{N}\rangle$ & $2.189(4)$ & $1.995(4)$ \\
$\Sigma\left({ }^{\circ}\right)$ & 17.3 & 16.8
\end{tabular}

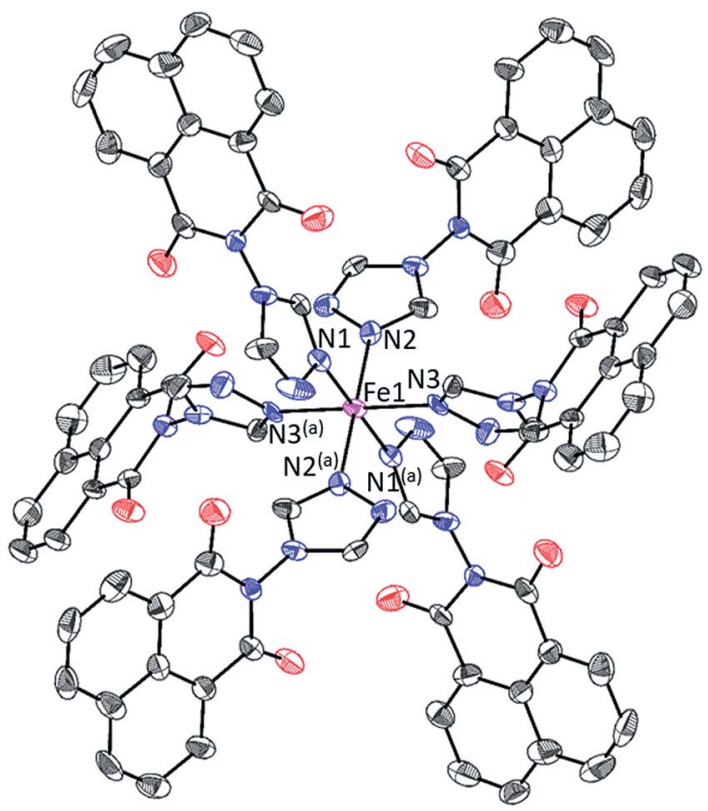

Fig. 2 Perspective ORTEP view of the molecular structure of the $\left[\mathrm{Fe}(\text { naph-trz })_{6}\right]^{2+}$ complex. Hydrogen atoms are omitted for the sake of clarity. Symmetry transformation used to generate equivalent atoms: (a) $-x,-y,-z$.
Table $\mathrm{S} 1 \dagger$ ). Selected $\mathrm{Fe}-\mathrm{N}$ bond lengths and $\mathrm{N}-\mathrm{Fe}-\mathrm{N}$ bond angles at both temperatures are gathered in Table S2. $\dagger$ Average $\mathrm{Fe}-\mathrm{N}$ distances and distortion parameters for $\mathrm{FeN}_{6}$ environment are listed in Table 1 for the two spin states.

The structure of $\mathbf{C 1}$ is built from one Fe(II) ion located on ( 0 0 0) special position, three naph-trz molecules acting as unidentate ligands, one (tcnsme) ${ }^{-}$anion and two solvent $\mathrm{CH}_{3} \mathrm{CN}$ molecules, all located on general positions. This asymmetric unit leads to the general formula [Fe(naph-trz) 6 ](tcnsme $)_{2} \cdot 4 \mathrm{CH}_{3} \mathrm{CN}$ (C1), in which the molecular structure consists of mononuclear complex of formula $\left[\mathrm{Fe}(\text { naph-trz })_{6}\right]^{2+}$ (Fig. 2), with the two (tcnsme) ${ }^{-}$anions acting as counterions to balance the charge of the complex. Examination of the $\mathrm{Fe}-\mathrm{N}$

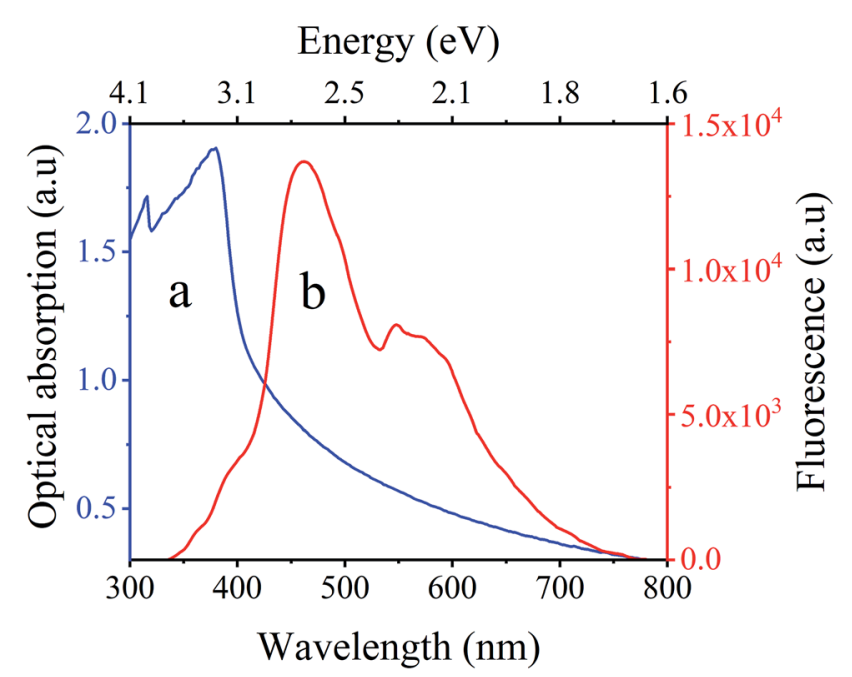

Fig. 3 Room temperature (a) optical absorption and (b) Fluorescence spectra of $\mathrm{C} 1$.

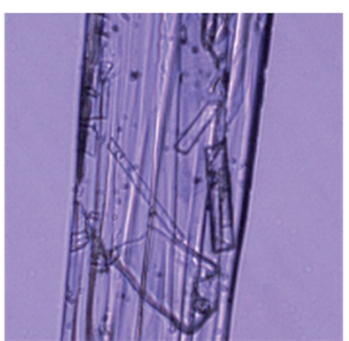

(a)

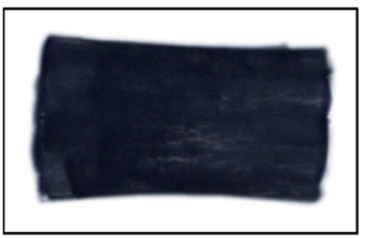

(c)

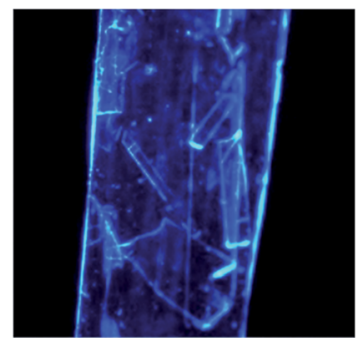

(b)

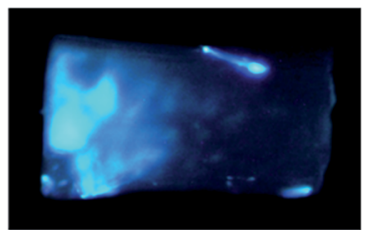

(d)
Fig. 4 Optical microscopy images at RT of (a) L1; (b) L1 under 320 nmlaser irradiation. (c) and (d) show the crystal C1 before and under irradiation with a $320 \mathrm{~nm}$-laser, respectively. The fluorescence of $\mathrm{L} 1$ is clearly stronger than that of $\mathrm{C} 1$. 

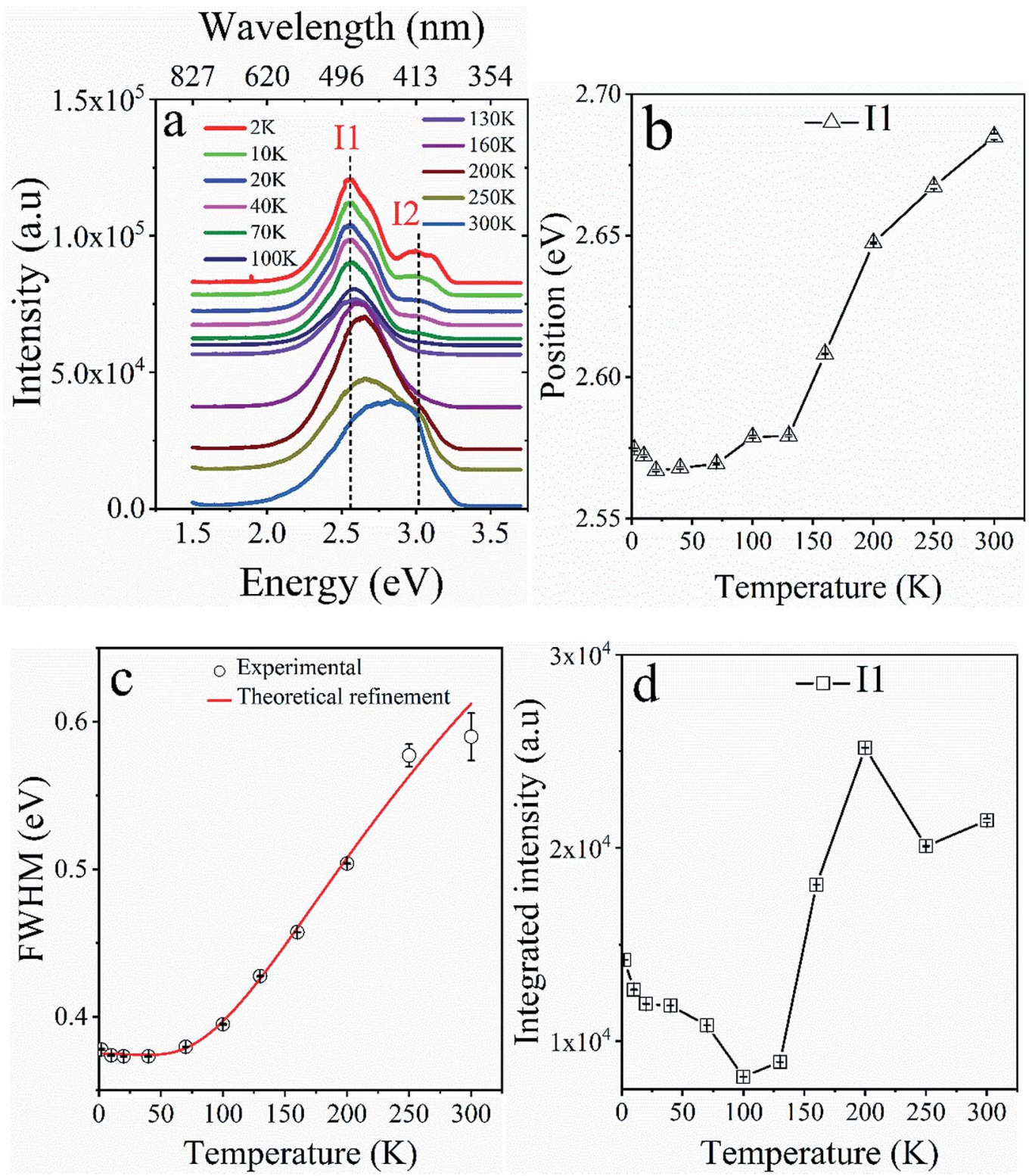

Fig. 5 (a) Temperature dependent of fluorescence properties of L1 over the range 2-300 K. Thermal evolution of the (b) position (in eV), (c) FWHM and (d) integrated intensity of the emission peak I1, showing significant changes around 150 and $200 \mathrm{~K}$.

bonds and the $\mathrm{N}-\mathrm{Fe}-\mathrm{N}$ bond angles, for the $\mathrm{Fe}^{\mathrm{II}}$ environments (Fe1), reveals that the central metal ion adopts a regular $\mathrm{FeN}_{6}$ octahedral geometry which is defined by six nitrogen atoms arising from six naph-trz molecules. The average values of the Fe-N distances at $296 \mathrm{~K}(2.189(4) \AA)$ and at $100 \mathrm{~K}(1.995(4) \AA)$ are in agreement with the presence of full HS and LS states, respectively. This observation is consistent with the presence of a HS $\leftrightarrow$ LS SCO transition, as revealed by the magnetic data (Fig. 1). The octahedral geometry of the metal center (Fe1) is relatively regular in the HS state, and therefore does not regularize further during the spin transition as revealed by the distortion parameter $\Sigma$ (Table 1 ) that is relatively small and remains almost unchanged between RT and $100 \mathrm{~K}$. This peculiar behavior, typical of constrained rigid systems, ${ }^{15}$ is probably induced by the presence of six sterically hindered heterocyclic bulky naph-trz ligands in the coordination sphere of the cationic complex. Examination of the crystal packing in $\mathbf{C 1}$ reveals that the shortest intermolecular distances correspond to the $\pi-\pi(\mathrm{C} \cdots \mathrm{C}=3.50-3.52 \AA)$ and to the $\mathrm{O} \cdots \mathrm{C}(3.20-3.30 \AA)$ contacts between the planar naph-trz heterocyclic ligands of adjacent complexes (see Fig. S14 $\dagger$ ). To understand how the unit cell parameters are affected by the SCO behavior, we have studied the temperature dependence of the lattice parameters of a single crystal of $\mathbf{C 1}$ in the temperature range 90-296 K. As expected, the unit cell parameters $(a, b, c)$ and volume $(V)$ as well as the $\beta$ angle show significant changes around the transition temperature (see Fig. S15 $\dagger$ ). Furthermore, the analysis of the thermal variation of the three metric parameters $(a, b, c)$ reveals, anisotropic changes around the transition temperature since, upon cooling from HS to the LS, the ac plane shows significant 

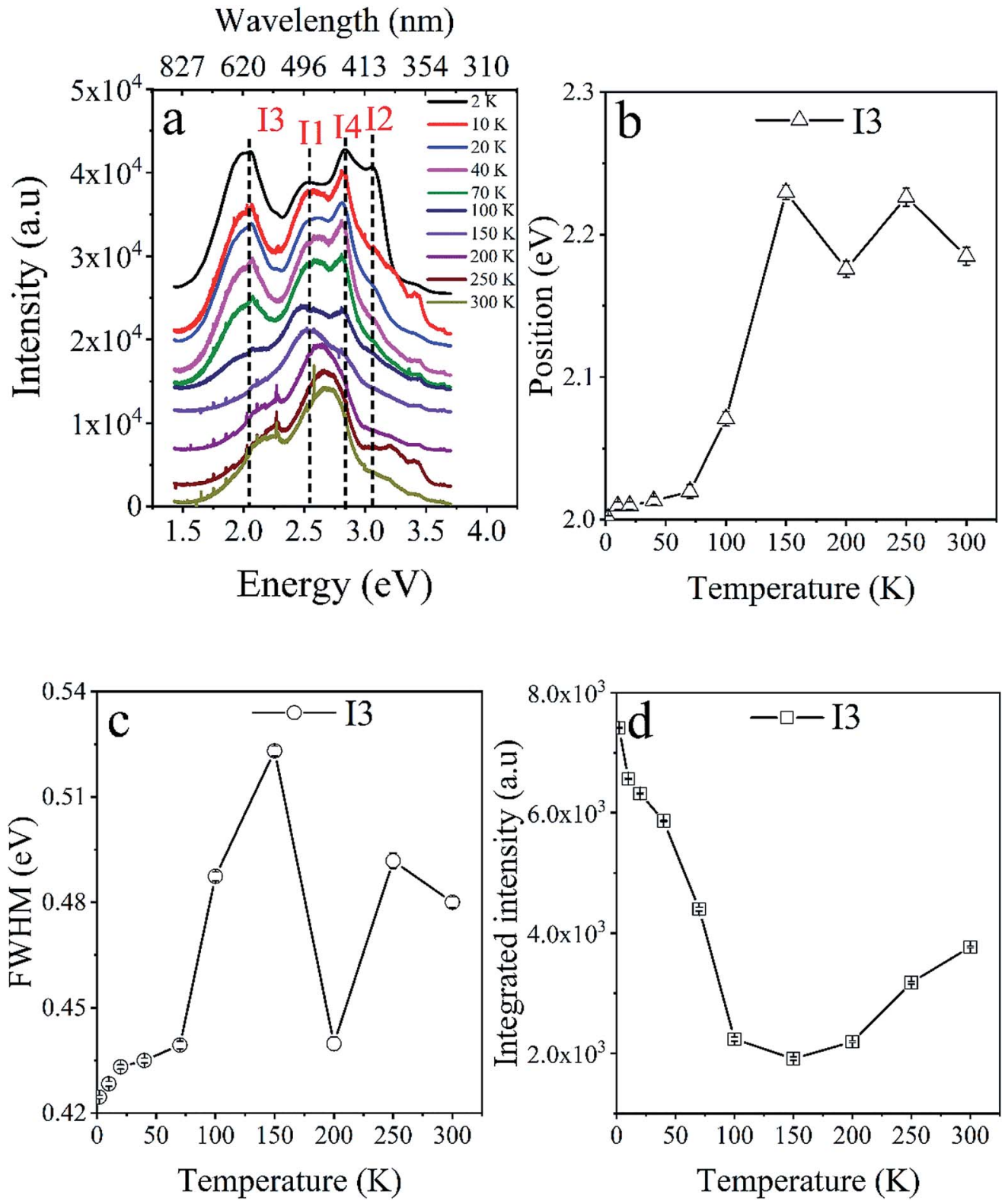

Fig. 6 (a) Fluorescence spectra of $\mathrm{C} 1$ over the temperature range 2-300 K showing the two extra components found (peaks 13 and 14 ) in comparison with the emission spectra of L1 shown in Fig. 5. Thermal dependence of (b) the position (in eV), (c) the FWHM and (d) the integrated intensity of 13 emission peak.

contraction along the two crystallographic directions, while the $b$ parameter increases (see Fig. S15†), a behavior that is frequently encountered in SCO solids. ${ }^{16}$ The impact of this overall change is an increase of unit cell volume by $3.5 \%$ upon LS to HS transition, in excellent agreement with usual crystallographic data of SCO materials. ${ }^{16}$

The apparent discrepancy between the transition temperatures obtained by magnetic and crystallographic measurements originates from the fact that magnetic data result from the average thermal response of a large number of SCO particles having different transition temperatures while the structural data give the thermal transition of one single crystal. To compare the crystallographic and magnetic data, we derived the corresponding HS fractions and plotted their variations as a function of the dimensionless parameter, $T / T_{1 / 2}$, where $T_{1 / 2}$ is the associated transition temperature (Fig. S16 $)$ ). While the procedure is simple for the magnetic curve, for the lattice parameters, $a, b, c$, it requires subtracting their natural thermal expansion which interferes with the spin transition, before to plot their relative variations. A good agreement is obtained between all these data, although the structural transition appears sharper with respect to the magnetic one. Similar conclusions are also found when comparing magnetic data with optical microscopy studies, performed on various SCO single crystals, which demonstrated that the transition temperature crucially depends on the crystal size and shape. ${ }^{17}$ 
Optical investigations of $\mathbf{C 1}$ were performed using optical absorption, fluorescence measurements (see ESI $\dagger$ ). Fig. 3a shows the RT optical absorption spectrum of C1 with a band centered at $380 \mathrm{~nm}$. Irradiation of $\mathbf{C 1}$ with a UV laser source at $325 \mathrm{~nm}$ at RT leads to the spectrum shown in Fig. 3, which has two bands peaking at $561 \mathrm{~nm}$ and $463 \mathrm{~nm}$ clearly confirming that $\mathbf{C 1}$ is fluorescent at RT. Moreover, optical microscopy images, recorded at RT, allow observation of the bluish fluorescent color of $\mathbf{C 1}$ as well as that of L1 at RT (Fig. 4).

Fluorescence properties of $\mathbf{L} 1$ were then investigated over the temperature range 2-300 K. The emission spectra of $\mathbf{L 1}$ as a function of temperature (Fig. $5 \mathrm{a}$ and $\mathrm{S} 17 \dagger$ ) show a complex structure with two well separated components centered at $486 \mathrm{~nm}$ (I1) and $412 \mathrm{~nm}$ (I2). The behavior of the spectra of $\mathbf{L 1}$ indicates that there are significant changes when $\mathbf{L 1}$ is heated from helium to ambient temperature. These changes manifest through a monotonous increase of the position and the full width at half maximum (FWHM) of I1 peak while its integrated intensity shows a singularity around $150 \mathrm{~K}$ (Fig. $5 \mathrm{~b}$ and c).

Moreover, important changes were also observed in the thermal behavior of the position, FWHM and the integrated intensity of I2 peak shown in Fig. S18. $\dagger$ Besides that, it is well known that the thermal dependence of the fluorescence line broadening may bring important information on the vibration/ rotations of the ligand. Hence, the FWHM of peak I1, is derived using a Lorentzian line shape fitting procedure, based on the following law,

$$
\Gamma(T)=\Gamma_{0}+\Gamma_{\mathrm{AC}} \times T+\Gamma_{\mathrm{LO}} \exp \left(-\frac{E_{\mathrm{LO}}}{k_{\mathrm{B}} T}\right),
$$

in which $\Gamma_{0}$ is the natural line width at $0 \mathrm{~K}$, the second term represents the broadening induced by acoustic phonons, and the third term accounts for the inhomogeneous broadening of the emission line width caused by optical vibrations. There, $\Gamma_{\mathrm{LO}}$ is the exciton-phonon coupling constant and $E_{\mathrm{LO}}$ is the optical phonon energy. The best fit gives, $\Gamma_{0}=375 \pm 4 \mathrm{meV}, \Gamma_{\mathrm{AC}}=1.2$ $\pm 0.1 \mathrm{meV} \mathrm{K}^{-1}, \Gamma_{\mathrm{LO}}=56 \pm 13 \mathrm{meV}$ and $E_{\mathrm{LO}}=28 \pm 3 \mathrm{meV}$. At low temperature, a strong exciton-phonon coupling takes place, governed by the relatively high exciton-phonon interaction constant $\Gamma_{\text {LO }}$ of $56 \mathrm{meV}$. In contrast, beyond $120 \mathrm{~K}$, considered as the high temperature region, the broadening of the luminescence peak is mainly caused by acoustic phonons ${ }^{\mathbf{1 8}}$ and shows a typical linear increase with a slope $1.2 \mathrm{meV} \mathrm{K}^{-1}$, whose value is in good agreement with usual findings in hybrid perovskites, for example. ${ }^{\mathbf{1 8}}$

The thermal response of the fluorescence spectra of $\mathbf{C 1}$ was investigated on single crystals in the same temperature region as for L1. The obtained results are summarized in Fig. 6 and S19-23. $\dagger$ A large part of the emission spectra of C1 is reminiscent of L1, where the main optical bands I1 and I2 are also observed, although they are slightly blue-shifted. Additionally, two extra contributions, not present in the fluorescence spectra of L1, emerge at $600 \mathrm{~nm}(\sim 2 \mathrm{eV})$ and $445 \mathrm{~nm}(2.78 \mathrm{eV})$ noted as peaks I3 and I4, respectively. These emission bands are assigned to the fluorescence of the ligand when coordinated to the Fe(II) ion in the SCO metal complex. This behavior confirms the contribution of the $\mathrm{Fe}(\mathrm{II})$ ions to the presence of the extra band $\mathrm{I} 3$ in the emission spectra of $\mathbf{C 1}$.

To further compare the behaviors of $\mathbf{L 1}$ and $\mathbf{C 1}$, we have plotted in Fig. 7 the thermal dependence of the position of peak I1 that is present in both compounds. A significant difference is noted on the behavior of the two peaks. Indeed, while peak I1 displays a monotonous blue-shift for sample L1, it exhibits a more complex temperature dependence in material $\mathbf{C 1}$, with the presence of a regime change around $100 \mathrm{~K}$, corresponding to the SCO transition region. Above $100 \mathrm{~K} \mathrm{L1}$ and $\mathbf{C 1}$ show the same behavior (Fig. 7).

The inspection of the thermal behaviour of the overall fluorescence of $\mathbf{C 1}$ (see Fig. S23†) shows that its maximum intensity is almost two times smaller than that of the emission of the organic ligand L1, at RT, but it still remains strong enough to be seen with the naked eye, (as displayed in the optical microscopy images shown in Fig. 4).

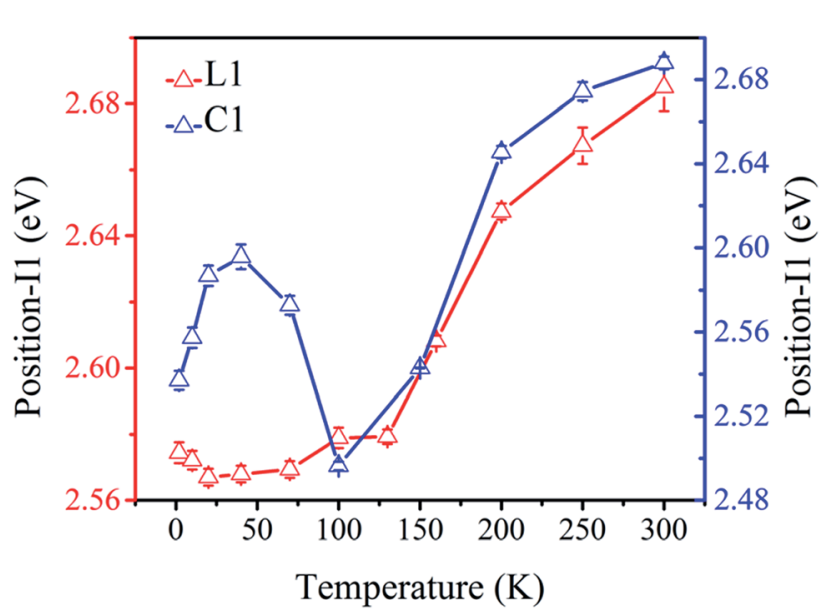

Fig. 7 Thermal-dependence of the positions (in eV) of peaks II in L1 (red) and C1 (blue) showing a significant difference in the thermal behavior of the emission at low-temperature region, i.e. in the LS region.

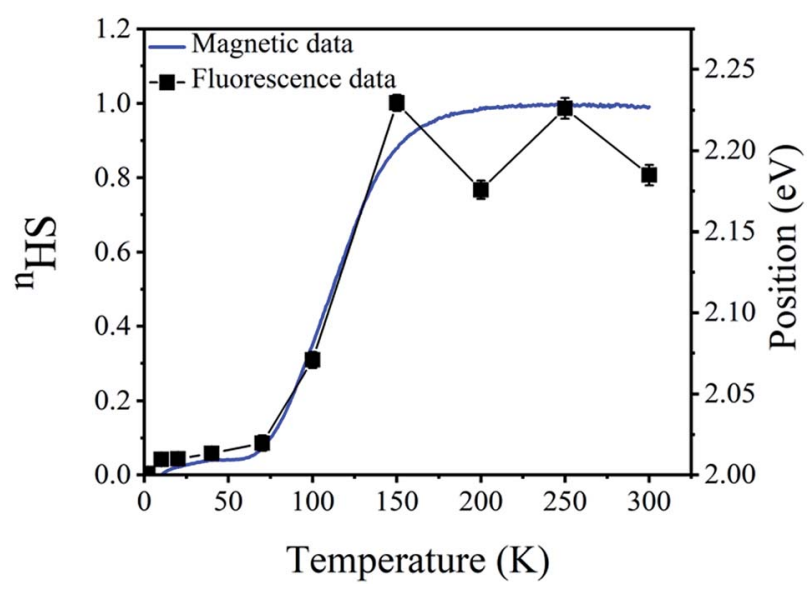

Fig. 8 Temperature dependence of peak 13 position in the emission spectra of the iron compound (black line with square symbol) in correlation with $n_{\mathrm{HS}}$ (blue line) derived from its magnetic properties. 
In order to study the global consistency of the experimental data of thermal dependence of the fluorescence properties of C1, we have correlated the results of the thermal-dependence of the HS fraction derived from magnetic measurements and those of the thermal evolution of the position of the extra peak I3 around $2 \mathrm{eV}(\sim 600 \mathrm{~nm})$ arising from the fluorescence response. The data, summarized in Fig. 8, show an excellent correlation, with the presence of a significant change in the temperature region $70-180 \mathrm{~K}$ where the thermal spin transition occurs in the magnetic response (Fig. 1a). These results clearly prove the existence of a direct synergy between the fluorescence properties of the complex and the spin transition of the central metal ion.

The spin transition causes an important change in the electronic density which is accompanied by a change of the $\mathrm{Fe}-$ ligand distances (structural reorganization), which undoubtedly affect the emission properties of the ligand. It is worth mentioning that at the present stage of the study it is not possible to bring more details about the coupling mechanism between the ligand and the SCO metal ion. We believe that DFT (Density Functional Theory) calculations, that we plan to study in a near future, will help to identify the intimate nature of this interaction. To further confirm the key role of the spin transition of the Fe(II) center, on the emission features of the complex C1, we have synthesized the homologous $\mathrm{Cu}$-containing compound $\left[\mathrm{Cu}(\text { naphtrz })_{6}\right](\text { tensme })_{2} \cdot 4 \mathrm{CH}_{3} \mathrm{CN}$ (C2). As expected, this sample does not show any spin transition. Fluorescence experiments performed on $\mathbf{C} 2$ at various temperatures (Fig. 9, S24 and 25†) clearly show that the characteristic optical band I3 is absent in $\mathbf{C} 2$ while the other bands look similar to I1 of L1 and I2 (of L1) or I4 (of C1), although slightly shifted and broadened, due to the effect of the d-electrons configuration of $\mathrm{Cu}$. This behavior confirms that the luminescence properties of

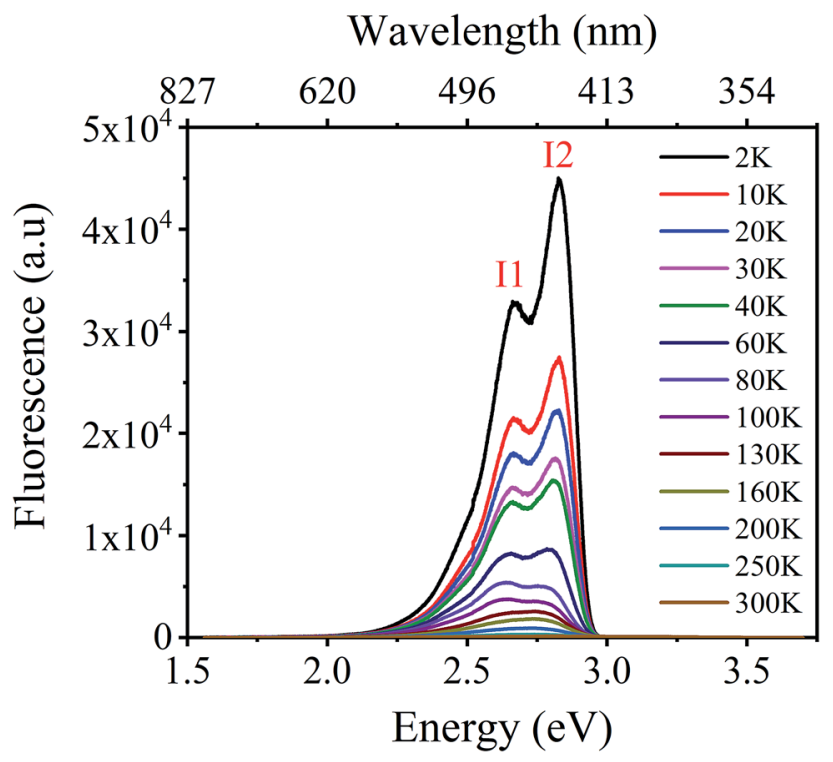

Fig. 9 Thermal-dependence of the fluorescence spectra of C2 showing only two peak contributions as for the ligand L1.
$\mathbf{L 1}$ are modified by the SCO behavior and therefore, constitutes a valuable and local probe of the spin transition phenomenon.

\section{Conclusions}

In summary, we have reported an iron(II) spin-crossover solid combing a spin transition and a strong fluorescence even at ambient temperature. Due to covalent character of the bonding between the six fluorescent ligands and the SCO metal center, the light emission spectra are significantly affected by the spintransition of the metal. As a result, and at least in this compound, the SCO transition can be revealed through the fluorescence spectroscopy. This property is of high importance, in particular, in the study of SCO nanoparticles for which the magnetic signal is too low to be studied in single objects, while the emission spectra can be still detected for small objects. Moreover, the thermal dependence of the intensity, the width and even more, the position of the luminescence peaks constitute an excellent local probe of temperature and mechanical constraints, particularly when reducing the size of the SCO particles. On the other hand, the close correlation observed in the present $\mathbf{C} \mathbf{1}$ complex between the gradual SCO and the luminescence behaviors, proves that the strong cooperativity is not needed for obtaining such synergetic multifunctional materials. Once again, this finding should be very useful for the design of fluorescent SCO thin films or nanoparticles, in which lower cooperativity is usually expected, for appropriate implementation in nanosized devices for enhanced sensing properties at the nanoscale. Finally, investigations of the photoinduced effects on the optical properties of the present SCO material are in progress as well as DFT calculations to get more insights about the quantum nature of the interrelationship of these two phenomena. Furthermore, in terms of innovative perspective to reach practical applications for the nano-switching devices, we are now designing systems based on new modified ligands involving similar triazole motif and fluorophore groups, separated by a flexible linker. Such ligands are able to favor the bridging coordination mode of the triazole motif at the origin of the formation $1 \mathrm{D}$ polymers, ${ }^{16 c}$ exhibiting strong cooperative effects, and from which we wish to reach the bistability of magnetic and fluorescence properties, around ambient temperature.

\section{Conflicts of interest}

The authors declare no conflict of interest.

\section{Acknowledgements}

The authors gratefully acknowledge the financial support of the CNRS, Université de Brest, Université de Versailles SaintQuentin-en-Yvelines, Université Paris Saclay, French Japan International Laboratory (LIA IM-LED), Spanish MINECO (project CTQ2017-87201-P AEU/FEDER UE) and Generalidad Valenciana (Prometeo Program). 


\section{Notes and references}

1 (a) S. Basak, P. Hui and R. Chandrasekar, Chem. Mater., 2013, 25, 3408-3413; (b) E. Coronado, J. R. Galán-Mascarós, M. Monrabal-Capilla, J. García-Martínez and P. PardoIbáñez, Adv. Mater., 2007, 19, 1359-1361; (c) M. Cavallini, Phys. Chem. Chem. Phys., 2012, 14, 11867-11876; (d) H. J. Shepherd, G. Molnár, W. Nicolazzi, L. Salmon and A. Bousseksou, Eur. J. Inorg. Chem., 2013, 2013, 653-661; (e) A. D. Naik, L. Stappers, J. Snauwaert, J. Fransaer and Y. Garcia, Small, 2010, 6, 2842-2846; (f) M. Cavallini, I. Bergenti, S. Milita, J. C. Kengne, D. Gentili, G. Ruani, I. Salitros, V. Meded and M. Ruben, Langmuir, 2011, 27, 4076-4081.

2 (a) A. Bousseksou, N. Negre, M. Goiran, L. Salmon, J. P. Tuchagues, M. L. Boillot, K. Boukheddaden and F. Varret, Eur. Phys. J. B, 2000, 13, 451-456; (b) M. A. Halcrow, Spin-Crossover Materials: Properties and Applications, John Wiley \& Sons Ltd, 2013; (c) J. Linares, E. Codjovi and Y. Garcia, Sensors, 2012, 12, 4479-4492; (d) A. Bousseksou, G. Molnar, L. Salmon and W. Nicolazzi, Chem. Soc. Rev., 2011, 40, 3313-3335; (e) P. Gutlich, A. B. Gaspar and Y. Garcia, Beilstein J. Org. Chem., 2013, 9, 342-391.

3 (a) P. Lloveras, E. Stern-Taulats, M. Barrio, J. L. Tamarit, S. Crossley, W. Li, V. Pomjakushin, A. Planes, L. Mañosa, N. D. Mathur and X. Moya, Nat. Commun., 2015, 6, 8801; (b) P. J. v. Ranke, Appl. Phys. Lett., 2017, 110, 181909.

4 X. Zhang, T. Palamarciuc, J.-F. Létard, P. Rosa, E. V. Lozada, F. Torres, L. G. Rosa, B. Doudin and P. A. Dowben, Chem. Commun., 2014, 50, 2255-2257.

5 (a) M. Hiroyuki, K. Keita and K. Nobuo, Chem. Lett., 2008, 37, 446-447; (b) C. Piguet, E. Rivara-Minten, G. Hopfgartner and J.-C. G. Bünzli, Helv. Chim. Acta, 1995, 78, 1541-1566; (c) C. Piguet, E. Rivara-Minten, G. Bernardinelli, J.-C. G. Bünzli and G. Hopfgartner, J. Chem. Soc., Dalton Trans., 1997, 421-434; (d) C. Edder, C. Piguet, J. C. G. Bünzli and G. Hopfgartner, Chem. - Eur. J., 2001, 7, 3014-3024.

6 (a) Y. Garcia, F. Robert, A. D. Naik, G. Zhou, B. Tinant, K. Robeyns, S. Michotte and L. Piraux, J. Am. Chem. Soc., 2011, 133, 15850-15853; (b) C.-F. Wang, R.-F. Li, X.-Y. Chen, R.-J. Wei, L.-S. Zheng and J. Tao, Angew. Chem., Int. Ed., 2015, 54, 1574-1577; (c) C. F. Wang, M. J. Sun, Q. J. Guo, Z. X. Cao, L. S. Zheng and J. Tao, Chem. Commun., 2016, 52, 14322-14325; (d) M. Hasegawa, F. Renz, T. Hara, Y. Kichuki, Y. Fukada, J. Okubo, T. Hoshi and W. Linert, Chem. Phys., 2002, 277, 21-30; (e) R. González-Prieto, B. Fleury, F. Schramm, G. Zoppellaro, R. Chandrasekar, O. Fuhr, S. Lebedkin, M. Kappes and M. Ruben, Dalton Trans., 2011, 40, 7564-7570; (f) J.-L. Wang, Q. Liu, X.-J. Lv, R.-L. Wang, C.-Y. Duan and T. Liu, Dalton Trans., 2016, 45, 18552-18558; $(g)$ B. Schäfer, T. Bauer, I. Faus, J. A. Wolny, F. Dahms, O. Fuhr, S. Lebedkin, H.-C. Wille, K. Schlage, K. Chevalier, F. Rupp, R. Diller, V. Schünemann, M. M. Kappes and M. Ruben,
Dalton Trans., 2017, 46, 2289-2302; (h) H. J. Shepherd, C. M. Quintero, G. Molnár, L. Salmon and A. Bousseksou, in Spin-Crossover Materials: Properties and Application, pp. 347-373, ed. M. A. Halcrow, John Wiley \& Sons Ltd, 2013.

7 (a) M. Matsuda, H. Isozaki and H. Tajima, Thin Solid Films, 2008, 517, 1465-1467; (b) K. Senthil Kumar and M. Ruben, Coord. Chem. Rev., 2017, 346, 176-205; (c) M. Masaki, I. Hikaru and T. Hiroyuki, Chem. Lett., 2008, 37, 374-375.

8 (a) J.-L. Wang, Q. Liu, Y.-S. Meng, X. Liu, H. Zheng, Q. Shi, C.-Y. Duan and T. Liu, Chem. Sci., 2018, 9, 2892-2897; (b) C.-F. Wang, G.-Y. Yang, Z.-S. Yao and J. Tao, Chem. - Eur. J., 2018, 24, 3218-3224.

9 (a) L. Salmon, G. Molnár, D. Zitouni, C. Quintero, C. Bergaud, J.-C. Micheau and A. Bousseksou, J. Mater. Chem., 2010, 20, 5499-5503; (b) S. Titos-Padilla, J. M. Herrera, X. W. Chen, J. J. Delgado and E. Colacio, Angew. Chem., Int. Ed., 2011, 50, 3290-3293.

10 C. A. Tovee, C. A. Kilner, J. A. Thomas and M. A. Halcrow, CrystEngComm, 2009, 11, 2069-2077.

11 (a) C. Lochenie, K. Schötz, F. Panzer, H. Kurz, B. Maier, F. Puchtler, S. Agarwal, A. Köhler and B. Weber, J. Am. Chem. Soc., 2018, 140, 700-709; (b) J. Yuan, S.-Q. Wu, M.-J. Liu, O. Sato and H.-Z. Kou, J. Am. Chem. Soc., 2018, 140, 9426-9433.

12 N. Zhang, A. B. Carter, A. J. Fitzpatrick and G. G. Morgan, J. Coord. Chem., 2016, 69, 2024-2037.

13 S. Lethu and J. Dubois, Eur. J. Org. Chem., 2011, 3920-3931. 14 M. Marchivie, P. Guionneau, J. F. Létard and D. Chasseau, Acta Crystallogr., Sect. B: Struct. Sci., 2005, 61, 25-28.

15 E. Milin, V. Patinec, S. Triki, E.-E. Bendeif, S. Pillet, M. Marchivie, G. Chastanet and K. Boukheddaden, Inorg. Chem., 2016, 55, 11652-11661.

16 (a) S. pillet, J. Hubsch and C. Lecomte, Eur. Phys. J. B, 2004, 38, 541-552; (b) M. Sy, F. Varret, K. Boukheddaden, G. Bouchez, J. Marrot and S. Kaizaki, Angew. Chem., Int. Ed., 2014, 53, 7539-7542; (c) N. Pittala, F. Thétiot, S. Triki, K. Boukheddaden, G. Chastanet and M. Marchivie, Chem. Mater., 2017, 29, 490-494.

17 (a) H. Fourati, G. Bouchez, M. Paez-Espejo, S. Triki and K. Boukheddaden, Crystals, 2019, 9, 46; (b) H. Fourati, E. Milin, A. Slimani, G. Chastanet, Y. Abid, S. Triki and K. Boukheddaden, Phys. Chem. Chem. Phys., 2018, 20, 10142-10154.

18 (a) A. Yangui, S. Pillet, A. Mlayah, A. Lusson, G. Bouchez, S. Triki, Y. Abid and K. Boukheddaden, J. Chem. Phys., 2015, 143, 224201; (b) A. Yangui, S. Pillet, E.-E. Bendeif, A. Lusson, S. Triki, Y. Abid and K. Boukheddaden, ACS Photonics, 2018, 5, 1599-1611; (c) A. Yangui, D. Garrot, J. S. Lauret, A. Lusson, G. Bouchez, E. Deleporte, S. Pillet, E. E. Bendeif, M. Castro, S. Triki, Y. Abid and K. Boukheddaden, J. Phys. Chem. C, 2015, 119, 2363823647; (d) M. Koubaa, T. Dammak, D. Garrot, M. Castro, E. Codjovi, A. Mlayah, Y. Abid and K. Boukheddaden, J. Appl. Phys., 2012, 111, 053521. 